\title{
Pacific
}

Journal of

Mathematics

\section{OPTIMAL APPROXIMATION CLASS FOR MULTIVARIATE BERNSTEIN OPERATORS}

\author{
ZeEv DitZian AND Xinlong Zhou
}




\section{OPTIMAL APPROXIMATION CLASS \\ FOR MULTIVARIATE BERNSTEIN OPERATORS}

\section{Z. Ditzian AND X. Zhou}

For the Bernstein polynomial approximation process on a simplex or a cube, the class of functions yielding optimal approximation will be given. That is, we will find the class of functions for which $\left\|B_{n} f-f\right\|_{C(S)}=O\left(n^{-1}\right)$ in terms of the behaviour of a certain $K$ functional. Moreover, this is done in the context of direct and converse results which yields an improvement on such results as well.

1. Introduction. For the simplex $S$ in $R^{d}$,

$$
S \equiv\left\{x=\left(x_{1}, \ldots, x_{d}\right): x_{i} \geq 0,|x| \equiv \sum_{i=1}^{d} x_{i} \leq 1\right\}
$$

the Bernstein polynomial approximation is given by

$$
B_{n} f=B_{n}(f, x) \equiv \sum_{\mu / n \in S} P_{n, \mu}(x) f\left(\frac{\mu}{n}\right), \quad x \in S,
$$

where $\mu=\left(m_{1}, \ldots, m_{d}\right)$ with $m_{i}$ integers, and

$$
\begin{aligned}
P_{n, \mu}(x) & \equiv \frac{n !}{\mu !(n-|\mu|) !} x^{\mu}(1-|x|)^{n-|\mu|}, \\
|x| & \equiv \sum_{i=1}^{d} x_{i}, \quad\left(|\mu| \equiv \sum_{i=1}^{d} m_{i}\right)
\end{aligned}
$$

with the convention

$$
\mu !=m_{1} ! \cdots m_{d} ! \quad \text { and } \quad x^{\mu}=x_{1}^{m_{1}} \cdots x_{d}^{m_{d}} .
$$

For the cube $Q$ in $R^{d}$,

$$
Q \equiv\left\{x=\left(x_{1}, \ldots, x_{d}\right): 0 \leq x_{i} \leq 1 \text { for } 1 \leq i \leq d\right\},
$$

the Bernstein polynomial approximation is given by

$$
\bar{B}_{n} f=\bar{B}_{n}(f, x) \equiv \sum_{\mu / n \in Q} \bar{P}_{n, \mu}(x) f\left(\frac{\mu}{n}\right), \quad x \in Q,
$$


where

$$
\begin{aligned}
\bar{P}_{n, \mu}(x) & \equiv \prod_{i=1}^{d} P_{n, m_{l}}\left(x_{i}\right) \text { and } \\
P_{n, l}(t) & \equiv \frac{n !}{l !(n-l) !} t^{l}(1-t)^{n-l} .
\end{aligned}
$$

We note that both (1.2) and (1.5) reduce to the classical Bernstein polynomials in case $d=1$.

The class of functions for which

$$
\left\|B_{n} f-f\right\|_{C(S)}=O\left(n^{-\alpha}\right) \quad\left(\text { or }\left\|\bar{B}_{n} f-f\right\|_{C(Q)}=O\left(n^{-\alpha}\right)\right)
$$

for $0<\alpha<1$ was determined by the first author [7]. Some additional articles were written in the past few years about the rate of approximation of $B_{n} f$ or $\bar{B}_{n} f$ to $f$ (see [8] and [13]). However, the determination of the class of functions for which the optimal rate of approximation is achieved, that is, $\left\|B_{n} f-f\right\|_{C(S)}=O\left(n^{-1}\right)$ or $\left\|\bar{B}_{n} f-f\right\|_{C(S)}=O\left(n^{-1}\right)$ eluded investigators of the subject (including [13], the billing of which in MR $89 \mathrm{k} 41007$ looked promising). It is clear that the rate $O\left(n^{-1}\right)$ is optimal as both $B_{n}$ and $\bar{B}_{n}$ satisfy conditions in [6] with $\sigma_{n}^{2}=n^{-1}$ and hence $\left\|B_{n} f-f\right\|_{C(S)}=o\left(n^{-1}\right)$ (or $\left\|\bar{B}_{n} f-f\right\|_{C(Q)}=o\left(n^{-1}\right)$ ) implies that $f$ is locally a solution of a certain elliptic partial differential equation given below in (2.2).

Recently, the rate of convergence of the related sequence of operators, that is, the Bernstein-Durrmeyer operators (see [1], [3] and [4]), was extensively and successfully investigated. One can only hope to match the success of the investigation of the Bernstein-Durrmeyer operators as those have properties like commutativity, self adjointness and simple expansion by orthogonal polynomials. Still, we were encouraged by the above mentioned success and, below, we have a saturation theorem for the rate of convergence of $B_{n} f-f$ (which is a much more difficult problem).

The result below will contain a characterization, that is, a necessary and sufficient condition on $f$ so that $\left\|B_{n} f-f\right\|_{C(S)}=O\left(n^{-1}\right)$ or $\left\|\bar{B}_{n} f-f\right\|_{C(Q)}=O\left(n^{-1}\right)$. The saturation result will be a consequence of a set of direct and converse inequalities, and thus, the generally difficult problem of unifying the direct-converse theorem with the saturation theorem is handled. 
It is hoped though, that stronger results will eventually emerge. We will explain the possibilities and make conjectures about further, more powerful results, in $\S 8$. We suspect that it will take quite some time as well as the introduction of new techniques before these conjectures are settled.

Here, we rely heavily on the investigation of best polynomial approximation on the simplex or on the cube [11, Chapter 12], an introduction of a three-term $K$-functional and on some recent results on multivariate Bernstein and Markov inequalities [8]. The use of these techniques, combined with some hard work and a new notation that overcomes the need to solve the problem in two dimensions first (see [6] and [2]), led us to the solution of the problems below on which we have worked for the last few years.

2. Further notations and the main result. We first introduce the elliptic differential operators that will be crucial in our investigation. For a polytope $A$ (that for us will be either the simplex $S$ of (1.1) or the cube $Q$ of (1.4)), we denote by $V_{A}$ the set of unit vectors in the directions of the edges of $A$ where $e$ and $-e$ are considered to be the same vector. We define, for a convex set $A$, a direction $\xi$, and a point $x \in A$,

$$
\begin{aligned}
\varphi_{\xi}(x)^{2} & \equiv \tilde{d}(\xi, x) \equiv \tilde{d}(A, \xi, x) \\
& \equiv \operatorname{Inf}_{\substack{x+\lambda \xi \notin A \\
\lambda>0}} d(x, x+\lambda \xi) \operatorname{Inf}_{\substack{x+\lambda \xi \notin A \\
\lambda<0}} d(x, x+\lambda \xi)
\end{aligned}
$$

where $d(x, y)$ is the Euclidean distance between $x$ and $y$ in $R^{d}$. The differential operators are now given by

$$
\begin{aligned}
& P(D) \equiv \sum_{\xi \in V_{S}} \tilde{d}(S, \xi, x)\left(\frac{\partial}{\partial \xi}\right)^{2} \\
& \bar{P}(D) \equiv \sum_{\xi \in V_{Q}} \tilde{d}(Q, \xi, x)\left(\frac{\partial}{\partial \xi}\right)^{2}
\end{aligned}
$$

where $S$ and $Q$ are given by (1.1) and (1.4), respectively. As $\tilde{d}\left(S, e_{i}, x\right)=x_{i}(1-|x|), \tilde{d}\left(S,\left(e_{i}-e_{j}\right) / \sqrt{2}, x\right)=2 x_{i} x_{j}, \tilde{d}\left(Q, e_{i}, x\right)$ $=x_{i}\left(1-x_{i}\right)$ and for $\xi=\left(e_{i}-e_{j}\right) / \sqrt{2}, \partial / \partial \xi=\frac{1}{\sqrt{2}}\left(\partial / \partial x_{i}-\partial / \partial x_{j}\right)$, 
we may write

$$
\begin{aligned}
P(D)= & \sum_{i=1}^{d} x_{i}(1-|x|)\left(\frac{\partial}{\partial x_{i}}\right)^{2} \\
& +\sum_{1 \leq i \leq j \leq d} x_{i} x_{j}\left(\frac{\partial}{\partial x_{i}}-\frac{\partial}{\partial x_{j}}\right)^{2} \\
\bar{P}(D)= & \sum_{i=1}^{d} x_{i}\left(1-x_{i}\right)\left(\frac{\partial}{\partial x_{i}}\right)^{2} .
\end{aligned}
$$

The $K$-functionals used in the present paper are now given by

$$
\begin{aligned}
K_{S}(f, t)=\operatorname{Inf}_{g \in C^{3}(S)}\left(\|f-g\|_{C(S)}\right. & +t^{2}\|P(D) g\|_{C(S)} \\
& \left.+t^{3} \operatorname{Sup}_{\xi \in V_{S}}\left\|\varphi_{\xi}^{3}(\partial / \partial \xi)^{3} g\right\|_{C(S)}\right)
\end{aligned}
$$

and

$$
\begin{aligned}
K_{Q}(f, t)=\operatorname{Inf}_{g \in C^{3}(Q)}\left(\|f-g\|_{C(Q)}\right. & +t^{2}\|\bar{P}(D) g\|_{C(Q)} \\
& \left.+t^{3} \operatorname{Sup}_{\xi \in V_{Q}}\left\|\varphi_{\xi}^{3}(\partial / \partial \xi)^{3} g\right\|_{C(Q)}\right)
\end{aligned}
$$

where $\varphi_{\xi}(x)$ of (2.4) and (2.5) is given by (2.1) with $A=S$ and $A=Q$, respectively.

The main result of this paper can now be stated.

TheOrem 2.1. For $B_{n} f$ and $K_{S}(f, t)$, given in (1.2) and (2.4), respectively, we have

$$
\left\|B_{n} f-f\right\|_{C(S)} \leq M\left(K_{S}\left(f, n^{-1 / 2}\right)+n^{-3 / 2}\|f\|_{C(S)}\right)
$$

and

$$
K_{S}\left(f, n^{-1 / 2}\right) \leq M n^{-3 / 2} \sum_{k=1}^{n} k^{1 / 2}\left\|B_{k} f-f\right\|_{C(S)}
$$

with $M$ independent of $f$ and $n$, and hence for $0<\alpha \leq 1$

$$
\left\|B_{n} f-f\right\|_{C(S)}=O\left(n^{-\alpha}\right) \Leftrightarrow K_{S}\left(f, n^{-1 / 2}\right)=O\left(n^{-\alpha}\right) \text {. }
$$

We observe that in the above theorem, (2.6) and (2.7) are the direct and converse results respectively and (2.8) for $\alpha=1$ is the saturation result.

The analogous result for the cube is given by: 
THEOREM 2.2. For $\bar{B}_{n} f$ and $K_{Q}(f, t)$ given by (1.5) and (2.5), respectively, we have

$$
\left\|\bar{B}_{n} f-f\right\|_{C(Q)} \leq M\left(K_{Q}\left(f, n^{1 / 2}\right)+n^{-3 / 2}\|f\|_{C(Q)}\right)
$$

and

$$
K_{Q}\left(f, n^{-1 / 2}\right) \leq M n^{-3 / 2} \sum_{k=1}^{n} k^{1 / 2}\left\|\bar{B}_{k} f-f\right\|_{C(Q)}
$$

with $M$ independent of $f$ and $n$, and hence for $0<\alpha \leq 1$

$$
\left\|\bar{B}_{n} f-f\right\|_{C(Q)}=O\left(n^{-\alpha}\right) \Leftrightarrow K_{Q}\left(f, n^{-1 / 2}\right)=O\left(n^{-\alpha}\right) .
$$

We will prove the more difficult Theorem 2.1 in more detail in $\S \S 4$, 5 and 6 and will comment on the necessary changes in the proof of Theorem 2.2 in $\S 7$. It is clear that (2.8) follows from (2.6) and (2.7) and that $(2.8)^{\prime}$ follows from $(2.6)^{\prime}$ and $(2.7)^{\prime}$.

3. Results about polynomials. This section will be dedicated to results on polynomials. Modifications of earlier results and rephrasing for the new notation are given for a somewhat more general situation than is needed for this paper. We hope these points will be useful elsewhere too.

We denote the set of polynomials of total degree $n$ by $\Pi_{n}$.

In [9], it was proved that for a bounded convex set $A$,

$$
\left\|\tilde{d}(A, \xi, \cdot)^{r / 2}(\partial / \partial \xi)^{r} P\right\|_{L_{p}(A)} \leq C n^{r}\|P\|_{L_{p}(A)} \quad \text { for } P \in \Pi_{n}
$$

where $r$ is an integer, $0<p \leq \infty, \xi \in R^{d},\|\xi\|=1$, and $\tilde{d}(A, \xi, x)$ is given in (2.1). For the set $S$, we can prove:

THEOREM 3.1. For $S$ given in (1.1), $0<p \leq \infty$, and $\xi \in V_{S}$,

$$
\left\|w(\cdot) \tilde{d}(\xi, \cdot)^{1 / 2}(\partial / \partial \xi) P\right\|_{L_{p}(S)} \leq C n\|w P\|_{L_{p}(S)}, \quad P \in \Pi_{n},
$$

where $w(x)=x_{1}^{\alpha_{1}} \cdots x_{d}^{\alpha_{d}}(1-|x|)^{\alpha_{d+1}}$ with $\alpha_{i} \geq 0$ for $p=\infty$ and $\alpha_{i}>-1 / p$ for $p<\infty$.

For Theorem 3.1, we may deduce the following result by repeating (3.2) using $\frac{\partial}{\partial \xi} P \in \Pi_{n}$ and the fact that $w(x)\left(\tilde{d}\left(\xi_{1}, x\right) \cdots \tilde{d}\left(\xi_{j}, x\right)\right)^{1 / 2}$ satisfies conditions on $w(x)$ in Theorem 3.1. 
Corollary 3.2. For $w(x), S$ and $p$ of Theorem 3.1 and $\xi_{i} \in V_{S}$, we have

$$
\begin{aligned}
& \left\|w(\cdot) \tilde{d}\left(\xi_{1}, \cdot\right)^{1 / 2} \cdots \tilde{d}\left(\xi_{k}, \cdot\right)^{1 / 2} \frac{\partial}{\partial \xi_{1}} \cdots \frac{\partial}{\partial \xi_{k}} P\right\|_{L_{p}(S)} \\
& \leq C n^{k}\|w P\|_{L_{p}(S)}, \quad P \in \Pi_{n} .
\end{aligned}
$$

Proof of Theorem 3.1. In fact, we only have to prove our result for $\xi=e_{1}$. If $\xi=e_{i}$, it is clear that renaming $i$ is sufficient. If $\xi=\left(e_{i}-e_{j}\right) / \sqrt{2}$, we write the polynomial $P(x)$ and the weight $w(x)$ as a polynomial and weight in $x_{r}$ for $r \neq j$ and $1-|x|$ for the $j$ variable. We now observe that $\partial / \partial \xi$ acts on the new variables like $2^{-1 / 2} \partial / \partial x_{i}$ and that $\tilde{d}(\xi, \cdot)^{1 / 2}$ contributes a factor of $\sqrt{2}$ multiplied by $d\left(e_{i}, \cdot\right)^{1 / 2}$ in the new variables. We note also that this transformation was used extensively in [7] and later in [8] and [2] for similar purposes. Using iterated integration and the notation $|\tilde{x}| \equiv x_{2}+\cdots+x_{d}$, it is clear that all that we have to show is

$$
\begin{aligned}
& \operatorname{Sup}_{0 \leq x_{1} \leq 1-|\tilde{x}|}\left|x_{1}^{\alpha+1 / 2}(1-|x|)^{\beta+1 / 2} \frac{\partial}{\partial x_{1}} P\left(x_{1}, \ldots, x_{d}\right)\right| \\
& \quad \leq C n \operatorname{Sup}_{0 \leq x_{1} \leq 1-|\tilde{x}|} x_{1}^{\alpha}(1-|x|)^{\beta}\left|P\left(x_{1}, \ldots, x_{d}\right)\right|
\end{aligned}
$$

and

$$
\begin{aligned}
& \int_{0}^{1-|\tilde{x}|}\left|x_{1}^{\alpha+1 / 2}(1-|x|)^{\beta+1 / 2} \frac{\partial}{\partial x_{1}} P\left(x_{1}, \ldots, x_{d}\right)\right|^{p} d x_{1} \\
& \leq C n^{p} \int_{0}^{1-|\tilde{x}|}\left|x_{1}^{\alpha}(1-|x|)^{\beta} \frac{\partial}{\partial x_{1}}\left(x_{1}, \ldots, x_{d}\right)\right|^{p} d x_{1}
\end{aligned}
$$

for $0<p<\infty$. We regard $x_{2}, \ldots, x_{d}$ as constants and make the change of variable $y=x_{1} /(1-|\tilde{x}|)$ and hence

$$
1-y=(1-|x|) /(1-|\tilde{x}|) \quad([0,1-|\tilde{x}|] \rightarrow[0,1])
$$

to obtain the result for $w^{*}(y) \frac{\partial}{\partial y} P^{*}(y)$ from [11, Theorem 8.4.7] for $1 \leq p \leq \infty$ and [14, Theorem 5] for $0<p<\infty$. (Both references have the interval $[-1,1]$ rather than $[0,1]$ as the underlying interval but that does not create any problems.)

We now recall a result about best polynomial approximation that will be crucial for the present paper. The rate of best polynomial approximation on a set $S$ is denoted by

$$
E_{n}(f)_{L_{p}(S)} \equiv \operatorname{Inf}_{P \in \Pi_{n}}\|f-P\|_{L_{p}(S)} .
$$


We further define $K$-functionals on the simple polytope $S$. We recall that a polytope (convex hull of finitely many points) in $R^{d}$ is simple if it has an interior point and each vertex is joined to other vertices by exactly $d$ edges.

(3.7) $K_{r, S}\left(f, t^{r}\right)_{p}$

$$
=\operatorname{Inf}_{g \in C^{r}(S)}\left(\|f-g\|_{L_{p}(S)}+t^{r} \operatorname{Sup}_{\xi \in V_{S}}\left\|\varphi_{\xi}^{r}(\partial / \partial \xi)^{r} g\right\|_{L_{p}(S)}\right)
$$

where $\varphi_{\xi}(x)$ is given by (2.1) (with $S=A$ ) and $V_{S}$ is the set of unit vectors in the directions of the edges of $S$. For much of this paper, $S$ can be regarded as a simplex or a cube which are simple polytopes. We now restate part of Theorem 12.2.3 of [11] in the following way which will be needed later.

Theorem 3.3 (Ditzian-Totik). Suppose $f \in L_{p}(S), 1 \leq p \leq \infty$ or $f \in C(S)$ (in which case the $L_{\infty}(S)$ norm is used). Suppose further, that $S$ is a simple polytope, (in particular, $S$ is a simple (1.1) or a cube (1.4)), $E_{n}(f)_{L_{p}(S)}$ is given by (3.6) and $K_{r, S}\left(f, t^{r}\right)_{p}$ is given by (3.7). Then

$$
E_{n}(f)_{L_{p}(S)} \leq C\left(K_{r, S}\left(f, n^{-r}\right)_{L_{p}(S)}+n^{-r}\|f\|_{L_{p}(S)}\right)
$$

and

$$
K_{r, S}\left(f, t^{r}\right)_{p} \leq M t^{r} \sum_{0 \leq k \leq 1 / t}(k+1)^{r-1} E_{k}(f)_{L_{p}(S)}
$$

Proof. The proof consists mainly of relating the present notation with the notation of [11, Chapter 12] to show that in fact, the theorem was proved there. We can write

$$
K_{r, S}\left(f, t^{r}\right)_{L_{p}(S)} \sim \operatorname{Sup}_{\xi \in V_{S}} \operatorname{Sup}_{0<h \leq t}\left\|\Delta_{h \varphi_{\xi} \xi}^{r} f\right\|_{L_{p}(S)} \equiv \bar{\omega}_{S}^{r}(f, t)_{p}
$$

where $\Delta_{\eta e}^{r}$ is given by

(3.11) $\Delta_{\eta e}^{r} f(x)$

$$
= \begin{cases}\sum_{k=0}^{r}\left(\begin{array}{l}
r \\
k
\end{array}\right)(-1)^{r} f(x+((r / 2)-k) \eta e), & x \pm \frac{r}{2} \eta e \in S, \\
0, & \text { otherwise. }\end{cases}
$$

The equivalence (3.10) follows the one-dimensional analogue [11, Chapter 2] and is actually implied in [11, Chapter 12] for an essentially 
identical situation. We now recall the modulus $\omega_{S}^{r}(f, t)_{p}$ from [11, p. 202] which is given by

$$
\omega_{S}^{r}(f, t)_{p} \equiv \operatorname{Sup}_{\xi \in V_{S}} \operatorname{Sup}_{0<h \leq t}\left\|\Delta_{h \tilde{d}_{S}(e, \cdot)^{1 / 2} \xi}^{r} f(\cdot)\right\|_{L_{p}(S)}
$$

where

$$
\tilde{d}_{S}(e, x) \equiv\left(\inf _{x+\lambda e \notin S} d(x, x+\lambda e)\right)\left(\max _{x+\lambda_{l} e \in S} d\left(x+\lambda_{1} e, x+\lambda_{2} e\right)\right) .
$$

(Note that (3.12) looks somewhat simpler but is exactly what is given in $[11$, p. 202].) We further observe that for $\tilde{d}(S, e, x)$ given in $(2.1)$, we have

$$
\frac{1}{2} \tilde{d}_{S}(e, x) \leq \tilde{d}(S, e, x) \leq \tilde{d}_{S}(e, x)
$$

and hence

$$
\omega_{S}^{r}(f, t / \sqrt{2}) \leq \bar{\omega}_{S}^{r}(f, t) \leq \omega_{S}^{r}(f, t)
$$

where $\bar{\omega}_{S}^{r}(f, t)$ and $\omega_{S}^{r}(f, t)$ are given in (3.10) and (3.12). We now note that

$$
\bar{\omega}_{S}^{r}(f, \alpha t)_{p} \leq C\left(\alpha^{r}+1\right) \bar{\omega}_{S}^{r}(f, t)_{p}
$$

which again follows from its analogue for one dimension [11, Theorem 4.1.2] or directly from (3.10) and is implied in [11, Chapter 12]. Combining (3.10), (3.13) and (3.14) with (12.2.3) and (12.2.4) of [11], we obtain (3.8) and (3.9). Note that the "proof" above consists mainly of matching and slightly modifying notations.

Further results which are corollaries of Theorem 3.3 will be used later for $p=\infty$ and where the simple polytope is a simplex or a cube.

THeORem 3.4. For $K_{r, S}\left(f, t^{r}\right)_{p}$ given by (3.7), $1 \leq p \leq \infty, r \in N$, and a simple polytope $S$, we have

$$
K_{r+1, S}\left(f, t^{r+1}\right)_{p} \leq C\left(K_{r, S}\left(f, t^{r}\right)_{p}+t^{r}\|f\|_{L_{p}(S)}\right)
$$

and

(3.16) $K_{r, S}\left(f, t^{r}\right)_{p}$

$$
\leq C\left[t^{r} \sum_{1 \leq k \leq 1 / t} k^{r-1} K_{r+1, S}\left(f, k^{-r-1}\right)_{p}+t^{r}\|f\|_{L_{p}(S)}\right] .
$$


Before we go into the proof which actually consists of substituting the results of Theorem 3.3 in an appropriate manner, we make some observations.

(a) Often the $K$-functional uses two norms rather than a norm and a seminorm for its definition. In this case, we define

$$
\begin{aligned}
K_{r, S}^{*}\left(f, t^{r}\right)_{p}=\operatorname{Inf}_{g \in C^{r}(S)}( & \|f-g\|_{L_{p}(S)} \\
& \left.+t^{r}\left(\|g\|_{L_{p}(S)}+\max _{\xi \in V_{S}}\left\|\varphi_{\xi}^{r}(\partial / \partial \xi)^{r} g\right\|_{L_{p}(S)}\right)\right)
\end{aligned}
$$

instead of (3.7) and we can rewrite (3.15) and (3.16) as

$$
K_{r+1, S}^{*}\left(f, t^{r+1}\right)_{p} \leq C K_{r, S}^{*}\left(f, t^{r}\right)_{p}
$$

and

$$
K_{r, S}^{*}\left(f, t^{r}\right)_{p} \leq C t^{r} \sum_{1 \leq k \leq 1 / t} k^{r-1} K_{r+1, S}^{*}\left(f, k^{-r-1}\right)_{p}
$$

which appear to be somewhat nicer but carry the same contents as (3.15) and (3.16).

(b) Using the equivalence in (3.10) and (3.13), we can restate (3.15) and (3.16) as

$$
\omega_{S}^{r+1}(f, t)_{p} \leq C\left(\omega_{S}^{r}(f, t)_{p}+t^{r}\|f\|_{L_{p}(S)}\right)
$$

and

$$
\omega_{S}^{r}(f, t)_{p} \leq C t^{r}\left(\int_{t}^{1} \frac{\omega_{S}^{r+1}(f, u)_{p}}{u^{r+1}} d u+\|f\|_{L_{p}(S)}\right)
$$

(The second inequality follows from $\int_{(k+1)^{-1}}^{k^{-1}} d u / u^{r+1} \sim k^{r-1}$.) We note that (3.16), (3.16) ${ }^{\prime}$ and (3.16)" are forms of the Marchaud-type inequality.

Proof of Theorem 3.4. We use (3.9) (with $r+1$ ) and then (3.8) (with r) to obtain

$$
\begin{aligned}
K_{r+1, S}\left(f, t^{r+1}\right)_{p} & \leq M t^{r+1} \sum_{1 \leq k \leq t^{-1}} k^{r} E_{k}(f)_{L_{p}(S)}+M t^{r+1}\|f\|_{L_{p}(S)} \\
& \leq M_{1} t^{r+1} \sum_{1 \leq k \leq t^{-1}} k^{r}\left[K_{r, S}\left(f, k^{-r}\right)_{p}+k^{-r}\|f\|_{L_{p}(S)}\right] .
\end{aligned}
$$


The definition of $K_{r, S}\left(f, t^{r}\right)_{p}$ in (3.7) implies

$$
K_{r, S}\left(f,(A t)^{r}\right)_{p} \leq A^{r} K_{r, S}\left(f, t^{r}\right)_{p} \text { for } A \geq 1
$$

which, with $A=1 / k t(k \leq 1 / t)$, completes the proof of (3.15). To prove (3.16), we just use (3.9) (with $r$ ) and then (3.8) (with $r+1)$.

4. Bernstein inequality for Bernstein polynomials. In this section, we will prove the Bernstein inequality estimate and other estimates of derivatives of Bernstein polynomials that will be crucial for our paper.

THeOREM 4.1. For $\xi \in V_{S}, B_{n} f$ defined by (1.2), $\nu=0,1$ and for $r=0,1,2, \ldots$, we have

$$
\left\|\varphi_{\xi}^{r+\nu}(\partial / \partial \xi)^{r+\nu} B_{n} f\right\|_{C(S)} \leq C n^{\nu / 2}\left\|\varphi_{\xi}^{r}(\partial / \partial \xi)^{r} f\right\|_{C(S)} .
$$

We observe that for $\nu=1,(4.1)$ yields the Bernstein-type inequality and for $\nu=0$ and $r>0,(4.1)$ yields the analogues of the inequalities in $\S 9.7$ of [11]. The reader should note that we save a substantial amount of work by not proving the inequality (4.1) for $\nu=2,3, \ldots$ which would have come in handy. The use of such estimates for higher $\nu$ is replaced in what follows by applying (4.1) to iterates of $B_{n} f$, that is, $B_{n}^{l} f$ and it will be shown that such estimates are sufficient for the proof of our main result.

Proof of Theorem 4.1. First, we recall from [7] the transformation that will allow us to consider (4.1) for $\xi=e_{1}$ only in which case $\partial / \partial \xi=\partial / \partial x_{1}$. It is clear that if $\xi=e_{i}$, we may just rename the coordinates. The transformation

$$
\left(u_{1}, \ldots, u_{d}\right)=T\left(x_{1}, \ldots, x_{d}\right), \quad \begin{aligned}
& u_{l}=x_{l} \text { for } l \neq j, \\
& u_{j}=1-x_{1}-\cdots-x_{d},
\end{aligned}
$$

introduced in [7], satisfies

$$
\begin{gathered}
T^{2}=I, \quad T: S \rightarrow S \text { onto, } \\
\frac{\partial}{\partial u_{l}}=\frac{\partial}{\partial x_{l}}-\frac{\partial}{\partial x_{j}} \quad \text { for } l \neq j, \quad \frac{\partial}{\partial u_{j}}=-\frac{\partial}{\partial x_{j}} ;
\end{gathered}
$$

it maps the point $e_{j}$ onto $(0, \ldots, 0)$ and a vector of $V_{S}$ onto a vector (not necessarily of Euclidean norm 1) in the direction of some edge of $S$. Also, we have for any $\xi \in V_{S}$,

$$
\left\|\tilde{d}(\xi, \cdot)^{r / 2}(\partial / \partial \xi)^{r} f(\cdot)\right\|=\left\|\tilde{d}(\eta, \cdot)^{r / 2}(\partial / \partial \eta)^{r} f_{T}(\cdot)\right\|
$$


where $f_{T}(u) \equiv f(T x), r \in N, \eta=T \xi /\|T \xi\|_{2}$ and $\|T \xi\|_{2}$ is the Euclidean norm in $R^{d}$. (Note that if $\xi=\left(e_{i}-e_{j}\right) / \sqrt{2}, \eta=e_{i}$ but $\tilde{d}(\xi, x)=2 x_{i} x_{j}=2 u_{i}\left(1-u_{1}-\cdots-u_{j}\right)=2 \tilde{d}(\eta, T x)$. $)$ For the Bernstein polynomials, we have

$$
B_{n}(f, x)=B_{n}\left(f_{T}, T x\right), \quad B_{n}(f, T x)=B_{n}\left(f_{T}, x\right) .
$$

Suppose we proved (4.1) for $\xi=e_{1}$ and hence for $\xi=e_{i}$; the above implies for $\xi=\left(e_{i}-e_{j}\right) / \sqrt{2}$ and $\eta=e_{i}$,

$$
\begin{aligned}
& \left\|\varphi_{\xi}(x)^{r+\nu}(\partial / \partial \xi)^{r+\nu} B_{n}(f, x)\right\| \\
& \quad=\left\|\varphi_{\eta}(T x)^{r+\nu}(\partial / \partial \eta)^{r+\nu} B_{n}\left(f_{T}, T x\right)\right\| \\
& \quad=\left\|\varphi_{\eta}(u)^{r+\nu}(\partial / \partial \eta)^{r+\nu} B_{n}\left(f_{T}, u\right)\right\| \\
& \quad \leq C n^{\nu / 2}\left\|\varphi_{\eta}(u)^{r}(\partial / \partial \eta)^{r} f_{T}(u)\right\| \\
& \quad=C n^{\nu / 2}\left\|\varphi_{\xi}(x)^{r}(\partial / \partial \xi)^{r} f(x)\right\| .
\end{aligned}
$$

Note that this type of argument is used repeatedly in the present paper and elsewhere and was given in detail here to utilize $T$ of [7] on the new, more efficient notations of the present paper. We now prove (4.1) for $\xi=e_{1}$.

For $P_{n, \beta}(x)$ given by $(1.3)$ and $P_{n, \beta}(x)=0$ for $\beta / n \notin S$, we have

$$
\frac{\partial}{\partial x_{1}} P_{n, \beta}(x)=n\left(P_{n-1, \beta-e_{1}}(x)-P_{n-1, \beta}(x)\right) \text {. }
$$

We now denote the forward difference by

(4.6) $\vec{\Delta}_{e h}^{r} f(x)$

$$
\equiv \begin{cases}\sum_{k=0}^{r}\left(\begin{array}{l}
r \\
k
\end{array}\right)(-1)^{r-k} f(x+k e h), & x, x+r e h \in S, \\
0, & \text { otherwise. }\end{cases}
$$

The routine calculation iterating the above implies now

$$
\begin{aligned}
\left(\frac{\partial}{\partial x_{1}}\right)^{r+\nu} B_{n}(f, x) & \\
= & n(n-1) \cdots(n-r+1) \\
& \cdot \sum_{\beta /(n-r) \in S} \vec{\Delta}_{e_{1} / n}^{r} f\left(\frac{\beta}{n}\right)\left(\frac{\partial}{\partial x_{1}}\right)^{\nu} P_{n-r, \beta}(x) .
\end{aligned}
$$

Recall that $\beta /(n-r) \in S$ implies $\beta / n, \beta / n+r e_{1} / n \in S$. We now write (recalling $\tilde{d}\left(e_{1}, x\right)=\varphi_{e_{1}}(x)^{2}=x_{1}(1-|x|)$ and $\beta=$ 
$\left.\left(k_{1}, \ldots, k_{d}\right)\right)$ for $\beta /(n-r) \in S$,

$$
\begin{aligned}
& \left|\vec{\Delta}_{e_{1} n^{-1}}^{r} f\left(\frac{\beta}{n}\right)\right| \\
& \quad=\int_{0}^{1 / n} \cdots \int_{0}^{1 / n}\left(\frac{\partial}{\partial x_{1}}\right)^{r} f\left(\frac{\beta}{n}+e_{1}\left(t_{1}+\cdots+t_{r}\right)\right) d t_{1} \cdots d t_{r} \\
& \quad \leq I(r) \int_{0}^{1 / n} \cdots \int_{0}^{1 / n}\left[\left(\frac{k_{1}}{n}+\sum_{i=1}^{r} t_{i}\right)\left(1-\frac{|\beta|}{n}-\sum_{i=1}^{r} t_{i}\right)\right]^{-r / 2} d t_{1} \cdots d t_{r} \\
& \quad \leq I(r)\left(\int_{0}^{1 / n}\left[\left(\frac{k_{1}}{n}+t\right)\left(1-\frac{|\beta|+r-1}{n}-t\right)\right]^{-1 / 2} d t\right)^{r}
\end{aligned}
$$

where $I(r)=\left\|\varphi_{e_{1}}^{r}\left(\partial / \partial x_{1}\right)^{r} f\right\|_{C(S)}$. For $\beta /(n-r) \in S$ (and hence $\left.0 \leq k_{1} \leq|\beta| \leq n-r\right)$, we have

$$
\begin{aligned}
\int_{0}^{1 / n} & \left(\frac{k_{1}}{n}+t\right)^{-1 / 2}\left(1-\frac{|\beta|+(r-1)}{n}-t\right)^{-1 / 2} d t \\
\leq & \left(1-\frac{|\beta|+(r-1)}{n}-\frac{1}{2 n}\right)^{-1 / 2} \int_{0}^{1 / 2 n}\left(\frac{k_{1}}{n}+t\right)^{-1 / 2} d t \\
& +\left(\frac{k_{1}}{n}+\frac{1}{2 n}\right)^{-1 / 2} \int_{1 / 2 n}^{1 / n}\left(1-\frac{|\beta|+r-1}{n}-t\right)^{-1 / 2} d t \\
\leq & C\left(\left(\frac{k_{1}+1}{n}\right)^{-1 / 2}\left(\frac{n-|\beta|-r+1}{n}\right)\right)^{-1 / 2} n^{-1} .
\end{aligned}
$$

Combining the above considerations, we have

$$
\begin{aligned}
& \tilde{d}\left(e_{1}, x\right)^{(r+\nu) / 2}\left|\left(\partial / \partial x_{1}\right)^{r+\nu} B_{n}(f, x)\right| \\
& \quad \leq C_{1}\left\|\tilde{d}\left(e_{1}, \cdot\right)^{r / 2}\left(\partial / \partial x_{1}\right)^{r} f(\cdot)\right\|_{C(S)} J_{n, \nu}(x)
\end{aligned}
$$

where

$$
\begin{aligned}
J_{n, \nu}(x)= & \left(x_{1}(1-|x|)\right)^{(r+\nu) / 2} \\
& \cdot \sum_{\beta /(n-r) \in S}\left(\frac{k_{1}+1}{n}\right)^{-r / 2}\left(1-\frac{|\beta|+r-1}{n}\right)^{-r / 2} \\
& \cdot\left|\left(\frac{\partial}{\partial x_{1}}\right)^{\nu} P_{n-r, \beta}(x)\right| .
\end{aligned}
$$

Using

$$
\frac{\partial}{\partial x_{1}} P_{l, \beta}(x)=\left(\frac{k_{1}(1-|x|)-(l-|\beta|) x_{1}}{x_{1}(1-|x|)}\right) P_{l, \beta}(x)
$$


for $l=n-r$ and the Cauchy-Schwarz inequality, we have

$$
\begin{aligned}
J_{n, \nu}(x)= & \left\{\left(x_{1}(1-|x|)\right)^{r}\right. \\
& \left.\cdot \sum_{\beta /(n-r) \in S}\left(\frac{k_{1}+1}{n}\right)^{-r}\left(\frac{n-\beta-r+1}{n}\right)^{-r} P_{n-r, \beta}(x)\right\}^{1 / 2} \\
& \times\left\{\left(x_{1}(1-|x|)\right)^{-\nu}\right. \\
& \left.\cdot \sum_{\beta /(n-r) \in S}\left(k_{1}(1-|x|)-(n-r-|\beta|) x_{1}\right)^{2 \nu} P_{n-r, \beta}(x)\right\}^{1 / 2} \\
\equiv & L_{n}(x)^{1 / 2} I_{n, \nu}(x)^{1 / 2} .
\end{aligned}
$$

For $\nu=0$, it is clear that $I_{n, \nu}(x)=1$. For $\nu=1$, one may calculate $I_{n, 1}(x)$ and obtain

$$
I_{n, 1}(x)=(n-r)\left(1-x_{2}-\cdots-x_{d}\right) .
$$

We omit this calculation as (3.8) of [4] implies $I_{n, 1}+J \leq(n-r) d$ where $J$ (given there) is positive and hence $I_{n, 1} \leq(n-r) d \leq C n$. To calculate $L_{n}(x)$, we follow Lemma 3.2 of [5] to write

$$
\begin{aligned}
x_{1}^{r}(1 & -|x|)^{r} P_{n-r, \beta}(x) \\
& =\frac{(n-r) !}{(n+r) !} \frac{(k+r) !}{k_{1} !} \frac{(n-|\beta|) !}{n !(|\beta|-r) !} \cdot P_{n+r, \beta+r e_{1}}(x)
\end{aligned}
$$

which implies that $L_{n}(x)$ is bounded as

$$
\frac{(n-r) !}{(n+r) !} \frac{\left(k_{1}+r\right) !}{k_{1} !} \frac{(n-|\beta|) !}{(n-|\beta|-r) !}\left(\frac{n}{k_{1}+1}\right)^{r}\left(\frac{n}{n-|\beta|-r+1}\right)^{r} \leq M
$$

with $M$ independent of $n$.

5. An estimate for Bernstein polynomial approximation of polynomials. In several articles (see [11, §9.3], [12] and [3]), extensive use is made of approximation of polynomials first which is later used to estimate approximation of other functions applying the result to polynomials of best approximation. This will be followed here for our present, more involved problem. We prove the necessary estimates on polynomials in this section and utilize the results in $\S 6$. It should be noted that we will be advancing the use of polynomials of best approximation somewhat further than earlier results and we hope that the techniques of this and the next section will prove fruitful as guidance for other situations as well.

The main result of this section is a strong Voronovskaja result for Bernstein polynomials on polynomials. 
TheOREM 5.1. For a polynomial $P \in \Pi_{m}, m \leq \sqrt{n}$, we have

$$
\begin{gathered}
\left|B_{n}(P, x)-P(x)-\frac{1}{2 n} \sum_{\xi \in V_{S}} \varphi_{\xi}(x)^{2}\left(\frac{\partial}{\partial \xi}\right)^{2} P(x)\right| \\
\leq M n^{-2} m^{4}\|P\|_{C(S)} .
\end{gathered}
$$

Proof. We follow [7] and divide the simplex $S$ into the regions $V_{i}=\left\{x \in S: x_{i}>1 / 2 d\right\}, i=1, \ldots, d$ and $V_{0}=\{x \in S: 1-|x|>$ $1 / 2 d\}$. Obviously, $\bigcup_{i=0}^{d} V_{i}=S$, and with the transformation $T$ of (4.2), we have $T: V_{j} \rightarrow V_{0}$ (onto) where we consider $j$ as generic. Furthermore, it is sufficient to prove (5.1) for $x \in V_{0}$. This follows as in the case $x \in V_{j}$, we can set $u=T x$ and $P_{T}(x) \equiv P(T x)$. From the result (5.1) for $u \in V_{0}$ and the polynomial $P_{T}$, we will obtain (5.1) for $x \in V_{j}$ and $P(x)$. To prove the above implication we write, for $x \in V_{j}, u=T x \in V_{0}$ and $\eta=T \xi /\|T \xi\|_{2}$,

$$
\begin{aligned}
& \left|B_{n}(P, x)-P(x)-\frac{1}{2 n} \sum_{\xi \in V_{S}} \varphi_{\xi}(x)^{2}\left(\frac{\partial}{\partial \xi}\right)^{2} P(x)\right| \\
& \quad=\left|B_{n}\left(P_{T}, u\right)-P_{T}(u)-\frac{1}{2 n} \sum_{\eta \in V_{S}} \varphi_{\eta}(u)^{2}\left(\frac{\partial}{\partial \eta}\right)^{2} P_{T}(u)\right| \\
& \quad \leq M n^{-2} m^{4}\left\|P_{T}\right\|_{C(S)}=M n^{-2} m^{4}\|P\|_{C(S)} .
\end{aligned}
$$

We further note that since the left-hand side of (5.1) is a polynomial of degree $m \leq \sqrt{n}$, it is sufficient to estimate it on $S_{1 / n}$ (or $S_{1 / n} \cap V_{0}$ ) given by

$$
S_{1 / n} \equiv\left\{x \in S: d\left(x, S^{c}\right) \geq \frac{1}{n}\right\} \text {. }
$$

This follows $S_{1 / m^{2}} \subset S_{1 / n}$ and hence Theorem 3.1 of [9] implies for $Q \in \Pi_{m}$,

$$
\|Q\|_{C(S)} \leq A\|Q\|_{C\left(S_{1 / n}\right)}
$$

We now need the following computational lemma which will be proved after the completion of the proof of Theorem 5.1.

LeMMA 5.2. For $\psi_{i}(x)=x_{i}, i=1, \ldots, d$, we have

(a) $B_{n}\left(\psi_{i}-x_{i}, x\right)=0$,

(b) $B_{n}\left(\left(\psi_{i}-x_{i}\right)^{2}, x\right)=x_{i}\left(1-x_{i}\right) / n$,

(c) $B_{n}\left(\left(\psi_{i}-x_{i}\right)^{3}, x\right)=\left(1 / n^{2}\right) x_{i}\left(1-x_{i}\right)\left(1-2 x_{i}\right)$, 
(d) $B_{n}\left(\left(\psi_{i}-x_{i}\right)^{2 r}, x\right) \leq C(A) n^{-r}\left(x_{i}\left(1-x_{i}\right)\right)^{r}$ for $A / n \leq x_{i} \leq$ $1-A / n$,

(e) $B_{n}\left(\left(\psi_{i}-x_{i}\right)\left(\psi_{j}-x_{j}\right), x\right)=-(1 / n) x_{i} x_{j}$ for $i \neq j$,

(f) $B_{n}\left(\left(\psi_{i}-x_{i}\right)\left(\psi_{j}-x_{j}\right)\left(\psi_{l}-x_{l}\right), x\right)=\left(2 / n^{2}\right) x_{i} x_{j} x_{l}$ for $i \neq j \neq$ $l \neq i$,

and

(g) $B_{n}\left(\left(\psi_{i}-x_{i}\right)\left(\psi_{j}-x_{j}\right)^{2}, x\right)=\left(1 / n^{2}\right)\left(2 x_{j}-1\right) x_{i} x_{j}$ for $i \neq j$.

We continue with the proof of Theorem 5.1 using Lemma 5.2. We expand $P\left(\frac{\beta}{n}\right)$ using Taylor's formula by

$$
\begin{aligned}
P\left(\frac{\beta}{n}\right)= & P(x)+\sum_{i=1}^{d}\left(\frac{k_{i}}{n}-x_{i}\right) \frac{\partial}{\partial x_{i}} P(x) \\
& +\frac{1}{2} \sum_{i, j=1}^{d}\left(\frac{k_{i}}{n}-x_{i}\right)\left(\frac{k_{j}}{n}-x_{j}\right) \frac{\partial^{2}}{\partial x_{i} \partial x_{j}} P(x) \\
& +\frac{1}{6} \sum_{i, j, l=1}^{d}\left(\frac{k_{i}}{n}-x_{i}\right)\left(\frac{k_{j}}{n}-x_{j}\right)\left(\frac{k_{l}}{n}-x_{l}\right) \\
& \cdot \frac{\partial^{3}}{\partial x_{i} \partial x_{j} \partial x_{l}} P(x)+R\left(\frac{\beta}{n}, x\right) \\
\equiv & P(x)+I_{1}\left(\frac{\beta}{n}, x\right)+I_{2}\left(\frac{\beta}{n}, x\right) \\
& +I_{3}\left(\frac{\beta}{n}, x\right)+R\left(\frac{\beta}{n}, x\right)
\end{aligned}
$$

where

$$
\begin{aligned}
& R\left(\frac{\beta}{n}, x\right)=\frac{1}{6} \int_{0}^{1} t^{3} F^{(4)}(t) d t, \\
& F(t)=P\left(\frac{\beta}{n}+t\left(x-\frac{\beta}{n}\right)\right) .
\end{aligned}
$$

As $B_{n}$ is a linear operator on functions of $\frac{\beta}{n}$ (where $x$ is considered a constant), we have

$$
B_{n}(P(\cdot), x)=B_{n}(P(x), x)+\sum_{r=1}^{3} B_{n}\left(I_{r}(\cdot, x), x\right)+B_{n}(R(\cdot, x), x) .
$$

Using $B_{n}(1, x)=1$, we have

$$
B_{n}(P(x), x)=P(x) \text {. }
$$


Using Lemma 5.1(a), we have $B_{n}\left(I_{1}(\cdot, x), x\right)=0$. Using Lemma 5.1(b) and (e) and a rearrangement of terms, we have

$$
\begin{aligned}
& B_{n}\left(I_{2}(\cdot, x), x\right) \\
& =\frac{1}{2 n}\left[\sum_{i=1}^{d} x_{i}\left(1-x_{i}\right)\left(\frac{\partial}{\partial x_{i}}\right)^{2} P(x)-2 \sum_{1 \leq i<j \leq d} x_{i} x_{j} \frac{\partial^{2}}{\partial x_{i} \partial x_{j}} P(x)\right] \\
& =\frac{1}{2 n}\left[\sum_{i=1}^{d} x_{i}(1-|x|)\left(\frac{\partial}{\partial x_{i}}\right)^{2}+\sum_{1 \leq i<j \leq d} x_{i} x_{j}\left(\frac{\partial}{\partial x_{i}}-\frac{\partial}{\partial x_{j}}\right)^{2}\right] P(x) \\
& =\frac{1}{2 n} \sum_{\xi \in V_{s}} \varphi_{\xi}(x)^{2}\left(\frac{\partial}{\partial \xi}\right)^{2} P(x) .
\end{aligned}
$$

Combining the above identities, it is evident that we will complete the proof when we estimate $\left|B_{n}\left(I_{3}(\cdot, x), x\right)\right|$ and $\left|B_{n}(R(\cdot, x), x)\right|$ by $C n^{-2} m^{4}\|P\|_{C(S)}$ for $x \in V_{0} \cap S_{1 / n}$.

To estimate $B_{n}\left(I_{3}(\cdot, x), x\right)$, we use (c), (f) and (g) of Lemma 5.2 to write

$$
\begin{aligned}
B_{n}\left(I_{3}(\cdot, x)\right) \leq & \frac{1}{6 n^{2}} \sum_{i=1}^{d} x_{i}\left(1-x_{i}\right)\left|1-2 x_{i}\right|\left|\left(\frac{\partial}{\partial x_{i}}\right)^{3} P(x)\right| \\
& +\frac{C}{n^{2}} \sum_{i<j<l} x_{i} x_{j} x_{l}\left|\frac{\partial}{\partial x_{i}} \frac{\partial}{\partial x_{j}} \frac{\partial}{\partial x_{l}} P(x)\right| \\
& +\frac{C}{n^{2}} \sum_{i \neq j}\left|2 x_{j}-1\right| x_{i} x_{j}\left|\frac{\partial^{2}}{\partial x_{j}^{2}} \frac{\partial}{\partial x_{i}} P(x)\right|
\end{aligned}
$$

Recalling that $\left|1-2 x_{i}\right| \leq 1$ and $0 \leq 1-x_{i} \leq 1$ for $x \in S$ and that $x_{i} \leq 2 d \varphi_{e_{i}}(x)^{2}$ for $x \in V_{0}$, we now have, for $P \in \Pi_{m}$,

$$
\begin{aligned}
& \left|x_{i}\left(1-x_{i}\right)\right| 1-2 x_{i}\left|\left(\frac{\partial}{\partial x_{i}}\right)^{3} P(x)\right| \leq 2 d\left|\varphi_{e_{i}}(x)^{2}\left(\frac{\partial}{\partial x_{i}}\right)^{2} \frac{\partial}{\partial x_{i}} P(x)\right| \\
& \leq C_{1} m^{2}\left\|\partial / \partial x_{i} P\right\|_{C(S)} \leq C_{2} m^{4}\|P\|_{C(S)} .
\end{aligned}
$$

For the second inequality above, we use Corollary 3.2 with $p=\infty$, $w(x)=1, \xi_{1}=\xi_{2}$ and $k=2$, and for the third inequality we use the multivariate Markov inequality

$$
\|(\partial / \partial \xi) P(x)\|_{C(S)} \leq C m^{2}\|P\|,
$$


proved in Theorem 4.1 of [9] (for instance). For $x \in V_{0}$, the estimates

$$
\begin{aligned}
x_{i} x_{j} x_{l}\left|\frac{\partial}{\partial x_{i}} \frac{\partial}{\partial x_{j}} \frac{\partial}{\partial x_{l}} P(x)\right| & \leq(2 d)^{3 / 2}\left\|\varphi_{e_{l}} \varphi_{e_{j}} \varphi_{e_{l}} \frac{\partial^{3}}{\partial x_{i} \partial x_{j} \partial x_{l}} P\right\| \\
& \leq C m^{3}\|P\|
\end{aligned}
$$

and

$$
\begin{aligned}
& x_{i} x_{j}\left|2 x_{j}-1\right|\left|\frac{\partial}{\partial x_{i}}\left(\frac{\partial}{\partial x_{j}}\right)^{2} P(x)\right| \\
& \leq(2 d)^{3 / 2}\left\|\varphi_{e_{i}} \varphi_{e_{j}}^{2} \frac{\partial}{\partial x_{i}}\left(\frac{\partial}{\partial x_{j}}\right)^{2} P\right\| \leq C m^{3}\|P\|
\end{aligned}
$$

follow from Corollary 3.2 with $p=\infty, w=1, k=3$ and $\xi_{l}$ chosen appropriately.

To estimate $B_{n}(R(\cdot, x), x)$, we recall that

(5.4) $F^{(4)}=\sum_{i_{1}=1}^{d} \cdots \sum_{i_{4}=1}^{d} \prod_{j=1}^{4}\left(x_{i_{j}}-\frac{k_{i_{j}}}{n}\right)$

$$
\times\left.\frac{\partial^{4}}{\partial x_{i_{1}} \partial x_{i_{2}} \partial x_{i_{3}} \partial x_{i_{4}}} P(\zeta)\right|_{\zeta=\beta / n+t(x-\beta / n)} .
$$

We now use the binomial expansion identity in the form

$$
\sum_{\beta / n \in S,|\beta| / n=1} P_{n, \beta}(x)=|x|^{n}
$$

to obtain, using the multivariate Markov inequality of [9, Theorem 4.1], for $x \in V_{0}$,

$$
\begin{aligned}
& \sum_{\beta / n \in S,|\beta| / n=1} P_{n, \beta}(x)\left|R\left(\frac{\beta}{n}, x\right)\right| \\
& \leq C|x|^{n} \max _{1 \leq i, \leq d}\left\|\frac{\partial}{\partial x_{i_{1}}} \cdots \frac{\partial}{\partial x_{i_{4}}} P\right\|_{C(S)} \\
& \leq C_{1}\left(1-\frac{1}{2 d}\right)^{n} m^{8}\|P\|_{C(S)} \\
& \leq C_{2} n^{-2} m^{4}\|P\|_{C(S)} .
\end{aligned}
$$

For $0 \leq t \leq 1, x \in S$ and $\beta / n \in S$, we have

$$
\frac{t\left|x_{i}-k_{i} / n\right|}{k_{i} / n+t\left(x_{i}-k_{i} / n\right)} \leq \frac{\left|x_{i}-k_{i} / n\right|}{x_{i}}
$$


which is trivial in case $x_{i}<k_{i} / n$ and follows from the fact that $t \alpha /(A+t \alpha)$ is increasing for $A>0, t>0$ and $\alpha>0$ in case $x_{i} \geq k_{i} / n$. Furthermore,

$$
\begin{aligned}
\frac{1}{1-(|\beta| / n+t(|x|-|\beta| / n))} & \leq \frac{1}{1-|x|}+\frac{1}{1-|\beta| / n} \\
& \leq C \frac{1}{1-|\beta| / n} \text { for } x \in V_{0} .
\end{aligned}
$$

In fact, we needed the estimate (5.6) because (5.7) is meaningless for $|\beta|=n$. Using (5.4) and (5.5), we only have to estimate

$$
\begin{aligned}
I= & \sum_{\beta / n \in S,|\beta| / n<1} P_{n, \beta}(x) \int_{0}^{1} t^{3}\left(\prod_{j=1}^{4}\left|x_{i_{j}}-\frac{k_{i_{j}}}{n}\right|\right) \\
& \times\left|\frac{\partial}{\partial x_{i_{1}}} \cdots \frac{\partial}{\partial x_{i_{4}}} P(\zeta)\right|_{\zeta=\beta / n+t(x-\beta / n)} \\
\leq & \|\left.\varphi_{e_{i_{1}}} \cdots \varphi_{e_{i_{4}}} \frac{\partial}{\partial x_{i_{1}}} \cdots \frac{\partial}{\partial x_{i_{4}}} P\right|_{\beta / n \in S,|\beta| / n<1} P_{n, \beta}(x) \\
& \times \int_{0}^{1} t^{3} \prod_{j=1}^{4}\left(\left|x_{i_{j}}-\frac{k_{i_{j}} \mid}{n}\right| / \varphi_{e_{i_{j}}}\left(\frac{\beta}{n}+t\left(x-\frac{\beta}{n}\right)\right)\right) d t .
\end{aligned}
$$

Using Corollary 3.2 (with $w=1$ ), (5.6) and (5.7), we have

$$
\begin{aligned}
& I \leq C m^{4}\|P\|_{C(S)} \sum_{\beta / n \in S,|\beta| / n<1} P_{n, \beta}(x)\left(\prod_{j=1}^{4} \frac{\left|x_{i_{j}}-k_{i_{j}} / n\right|}{x_{i_{j}}^{1 / 2}}\right) \frac{1}{(1-|\beta| / n)^{2}} \\
& \leq C m^{4}\|P\|_{C(S)} \prod_{j=1}^{4} \frac{1}{x_{i_{j}}^{1 / 2}}\left(\sum_{\beta / n \in S,|\beta| / n<1} P_{n, \beta}(x) \frac{\mid x_{i_{j}}-k_{i_{j} /\left.n\right|^{4}}}{(1-|\beta| / n)^{2}}\right)^{1 / 4} \\
& \leq C m^{4}\|P\|_{C(S)} \prod_{j=1}^{4} \frac{1}{x_{i_{j}^{1 / 2}}^{1 / 2}}\left(\sum_{\beta / n \in S} P_{n, \beta}(x)\left|x_{i_{j}}-\frac{k_{i_{j}}}{n}\right|^{8}\right)^{1 / 8} \\
& \cdot\left(\sum_{\beta / n \in S,|\beta| / n<1} \frac{P_{n, \beta}(x)}{(1-|\beta| / n)^{4}}\right)^{1 / 8} .
\end{aligned}
$$

We now use (d) of Lemma 5.2 with $r=4$, where we need to recall that $x \in S_{4 n}$ implies $x_{i} \geq 1 / d n(A=1 / d)$, and the estimate $1-x_{i} \leq 1$, 
to obtain

$$
\frac{1}{x_{i_{j}}^{1 / 2}}\left(\sum_{\beta / n \in S} P_{n, \beta}(x)\left|x_{i_{j}}-\frac{k_{i_{j}}}{n}\right|^{8}\right)^{1 / 8} \leq \frac{C}{n^{1 / 2}} .
$$

For $|\beta|<n$, we have

$$
\begin{aligned}
& (1-|x|)^{4} P_{n, \beta}(x)\left(1-\frac{|\beta|}{n}\right)^{-4} \\
& \quad \leq \frac{n !}{(n+4) !} \frac{(n+4-|\beta|) !}{(n-|\beta|) !} \frac{n^{4}}{(n-|\beta|)^{4}} P_{n+4, \beta}(x) \leq M P_{n+4, \beta}(x)
\end{aligned}
$$

where $M$ is independent of $n$ and hence for $x \in V_{0}$,

$$
\sum_{\beta / n \in S, \beta / n<1} P_{n, \beta}(x)\left(1-\frac{|\beta|}{n}\right)^{-4} \leq M_{1}
$$

which, together with the above, completes the proof of Theorem 5.1 pending the proof of Lemma 5.2.

Proof of Lemma 5.2. Parts (a), (b), (c) and (d) follow from summing first on the other indices $(\neq i)$ and observing that what we have is the $r$ th moment of the univariate Bernstein polynomial in $x_{i}$. The exact expressions (a), (b) and (c) are known from Lemma 9.4.3 of [11] (for example) and the estimate (d) from Lemma 9.4.4 of [11]. We now calculate

$$
\begin{aligned}
B_{n}\left(\psi_{i} \psi_{j}, x\right) & =\left.\frac{x_{i} x_{j}}{n^{2}} \frac{\partial}{\partial x_{i}} \frac{\partial}{\partial x_{j}}\left(z+\sum_{i=1}^{d} x_{i}\right)^{n}\right|_{z=1-|x|} \\
& =x_{i} x_{j}\left(1-\frac{1}{n}\right) \text { for } i \neq j, \\
B_{n}\left(\psi_{i} \psi_{j} \psi_{l}, x\right) & =\left.\frac{x_{i} x_{j} x_{l}}{n^{2}} \frac{\partial}{\partial x_{i}} \frac{\partial}{\partial x_{j}} \frac{\partial}{\partial x_{l}}\left(z+\sum_{i=1}^{d} x_{i}\right)^{n}\right|_{z=1-|x|} \\
& =x_{i} x_{j} x_{l}\left(1-\frac{1}{n}\right)\left(1-\frac{2}{n}\right) \text { for } i \neq j \neq l \neq i,
\end{aligned}
$$

and

$$
\begin{aligned}
B_{n}\left(\psi_{i} \psi_{j}^{2}, x\right) & =\left.\frac{x_{i} x_{j}}{n^{3}} \frac{\partial}{\partial x_{j}} x_{j} \frac{\partial}{\partial x_{j}} \frac{\partial}{\partial x_{i}}\left(z+\sum_{i=1}^{d} x_{i}\right)^{n}\right|_{z=1-|x|} \\
& =x_{i} x_{j}^{2}\left(1-\frac{1}{n}\right) \cdot\left(1-\frac{2}{n}\right)+\frac{x_{i} x_{j}}{n}\left(1-\frac{1}{n}\right) \text { for } i \neq j
\end{aligned}
$$

Simple arithmetic now implies (e), (f) and (g). 
6. Proof of the main result. First, we apply the strong Voronovskaja estimate for polynomials given in Theorem 5.1 to polynomials of best approximation to a function $f$. In this section, \|\|$=\|\|_{C(S)}$, $K_{r, S}\left(f, t^{r}\right)=K_{r, S}\left(f, t^{r}\right)_{\infty}$ and $E_{n}(f)=E_{n}(f)_{C(S)}$ with a simplex $S$ of (1.1).

Theorem 6.1. For $f \in C(S)$ and $P_{m} \in \Pi_{m}(m=[\sqrt{n}])$ satisfying $\left\|P_{m}-f\right\| \leq M E_{m}(f)$ and for $K_{3, S}\left(f, t^{3}\right)$ given by (3.7), we have

$$
\begin{aligned}
& \left\|B_{n} P_{m}-P_{m}-(2 n)^{-1} P(D) P_{m}\right\| \\
& \quad \leq\left(K_{3, S}\left(f, n^{-3 / 2}\right)+n^{-3 / 2}\|f\|\right) .
\end{aligned}
$$

Proof. We choose $P_{j}, P_{j} \in \Pi_{j}$, satisfying $\left\|P_{j}-f\right\| \leq M E_{j}(f)$ and expand $P_{m}$ by

$$
\begin{aligned}
P_{m}=P_{m}-P_{2^{l}}+\sum_{j=1}^{l}\left(P_{2^{j}}-P_{2^{j-1}}\right) & P_{1}, \\
l & =\max \left\{j: 2^{j}<m\right\} .
\end{aligned}
$$

We recall $B_{n} P_{1}-P_{1}=P(D) P_{1}=0$ and utilize Theorem 5.1 to write (for $m=[\sqrt{n}]$ )

$$
\begin{aligned}
I(n) & =\left\|B_{n} P_{m}-P_{m}-(2 n)^{-1} P(D) P_{m}\right\| \\
& \leq C_{1} n^{-2}\left(m^{4}\left\|P_{m}-P_{2^{l}}\right\|+\sum_{j=1}^{l} 2^{4 j}\left\|P_{2^{\prime}}-P_{2^{j-1}}\right\|\right) \\
& \leq C_{2} n^{-2}\left(m^{4} E_{2^{l}}(f)+\sum_{j=1}^{l} 2^{4 j} E_{2^{j-1}}(f)\right) .
\end{aligned}
$$

Applying now (3.8) of Theorem 3.3, we have

$$
I(n) \leq C_{3} n^{-2} \sum_{j=0}^{l} 2^{4 j}\left(K_{3, S}\left(f, 2^{-3 j}\right)+2^{-3 j}\|f\|\right) .
$$

Using the definition of $K_{3, S}\left(f, t^{3}\right)$, we have

$$
K_{3, S}\left(f,(A t)^{3}\right) \leq A^{3} K_{3, S}\left(f, t^{3}\right) \quad \text { for } A \geq 1
$$


We now choose in (6.3) $t=2^{-l}$, with $l$ of (6.2), and $A=2^{l-j}$ and then $t=n^{-1 / 2}$ and $A=2^{-l} / n^{1 / 2}$ and obtain

$$
\begin{aligned}
I(n) & \leq C_{4} n^{-2} \sum_{j=0}^{l} 2^{4 j} 2^{-3 j}\left(2^{3 l} K_{3, S}\left(f, 2^{-3 l}\right)+\|f\|\right) \\
& \leq C_{5}\left(n^{-2} 2^{4 l}\left(K_{3, s}\left(f, 2^{-3 l}\right)+n^{-3 / 2}\|f\|\right)\right) \\
& \leq C\left(K_{3, S}\left(f, n^{-1 / 2}\right)+n^{-3 / 2}\|f\|\right) .
\end{aligned}
$$

We are now able to prove the main result.

Proof of Theorem 2.1. Using the definition of $K_{S}(f, t)$ in (2.4), there exists a function $g \in C^{3}(S)$ such that

$$
\begin{aligned}
\| f- & g\left\|+n^{-1}\right\| P(D) g \| \\
& +n^{-3 / 2} \max _{\xi \in V_{S}}\left\|\varphi_{\xi}^{3}(\partial / \partial \xi)^{3} g\right\| \leq 2 K_{S}\left(f, n^{-1 / 2}\right) .
\end{aligned}
$$

We observe that the $K$-functional $K_{3, S}\left(f, t^{3}\right)$ given by (3.7) satisfies $K_{3, S}\left(f, n^{-3 / 2}\right) \leq K_{S}\left(f, n^{-1 / 2}\right)$ and $K_{3, S}\left(g, n^{-3 / 2}\right) \leq 2 K_{S}\left(f, n^{-1 / 2}\right)$ for all $f \in C(S)$ and $g \in C^{3}(S)$ satisfying (6.4). For a polynomial $P_{m} \equiv P_{m}(g)$ satisfying

$$
\left\|P_{m}-g\right\|=E_{m}(g), \quad P_{m} \in \Pi_{m} \quad \text { and } \quad m=[\sqrt{n}],
$$

we have, using Theorem 6.1,

$$
\begin{aligned}
\left\|B_{n} P_{m}-P_{m}\right\| \leq & \left\|B_{n} P_{m}-P_{m}-(2 n)^{-1} P(D) P_{m}\right\| \\
& +(2 n)^{-1}\left\|P(D) P_{m}\right\| \\
\leq & C\left(K_{3, S}\left(g, n^{-3 / 2}\right)+n^{-3 / 2}\|g\|+n^{-1}\left\|P(D) P_{m}\right\|\right) \\
\leq & C_{1}\left(K_{S}\left(f, n^{-1 / 2}\right)+n^{-1}\left\|P(D) P_{m}\right\|+n^{-3 / 2}\|f\|\right) .
\end{aligned}
$$

Furthermore,

$$
\begin{aligned}
\left\|P_{m}-g\right\| & \leq M E_{m}(g) \leq M_{1}\left(K_{3, S}\left(g, m^{-3}\right)+m^{-3}\|g\|\right) \\
& \leq M_{2}\left(K_{S}\left(f, n^{-1 / 2}\right)+n^{-3 / 2}\|f\|\right)
\end{aligned}
$$

and as $g$ satisfies (6.4),

$$
\left\|P_{m}-f\right\| \leq M_{3}\left(K_{S}\left(f, n^{-1 / 2}\right)+n^{-3 / 2}\|f\|\right) .
$$

Hence,

$$
\begin{aligned}
\left\|B_{n} f-f\right\| & \leq\left\|B_{n} P_{m}-P_{m}\right\|+2 M_{3}\left(K_{S}\left(f, n^{-1 / 2}\right)+n^{-3 / 2}\|f\|\right) \\
& \leq C_{2}\left(K_{S}\left(f, n^{-1 / 2}\right)+n^{-1}\left\|P(D) P_{m}\right\|+n^{-3 / 2}\|f\|\right) .
\end{aligned}
$$


To prove (2.6), it remains only to show that

$$
n^{-1}\left\|P(D) P_{m}\right\| \leq L\left(K_{S}\left(f, n^{-1 / 2}\right)+n^{-3 / 2}\|f\|\right) .
$$

Following (6.4), it is sufficient to estimate $n^{-1}\left\|P(D)\left(g-P_{m}\right)\right\|$. We write

$$
\text { (6.7) } g-P_{m}=P_{2^{l}}-P_{m}+\sum_{j=l}^{\infty}\left(P_{2^{l+1}}-P_{2^{j}}\right), \quad l=\min \left\{j: 2^{j}>m\right\}
$$

and use Corollary 3.2 to obtain

$$
\begin{aligned}
n^{-1}\left\|P(D)\left(g-P_{m}\right)\right\| \leq L_{1} n^{-1}\left(m^{2} E_{m}(g)+\sum_{j=1}^{\infty} 2^{(j+1) 2} E_{2^{j}}(g)\right) & \\
\leq L_{2} n^{-1}\left(m^{2} K_{3, S}\left(g, m^{-3}\right)+\right. & \sum_{j=1}^{\infty} 2^{2 j} K_{3, S}\left(g, 2^{-3 j}\right) \\
& \left.+\left(m^{2} m^{-3}+\sum_{j=l}^{\infty} 2^{-j}\right)\|g\|\right) .
\end{aligned}
$$

We recall from (3.7) and (6.4) that

$$
K_{3, S}\left(g, t^{3}\right) \leq t^{3} \operatorname{Sup}_{\xi \in V_{S}}\left\|\varphi_{\xi}^{3}(\partial / \partial \xi)^{3} g\right\| \leq 2 t^{3} n^{3 / 2} K_{S}\left(f, n^{-1 / 2}\right)
$$

and hence with the choices $t=m^{-1}$ and $t=2^{-j}$, we obtain (6.5), and the proof of (2.6) is complete.

To prove (2.7), we define

$$
B_{k}^{r}(f, x) \equiv B_{k}\left(B_{k}^{r-1} f, x\right), \quad B_{k}^{1}(f, x)=B_{k}(f, x)
$$

and obtain, using the definition of $K_{4, S}\left(f, t^{4}\right)$,

$$
K_{4, S}\left(f, t^{4}\right) \leq\left\|f-B_{k}^{4} f\right\|+t^{4} \operatorname{Sup}_{\xi \in V_{S}}\left\|\varphi_{S}^{4}(\partial / \partial \xi)^{4} B_{k}^{4} f\right\| .
$$

The elementary estimate yields

$$
\left\|f-B_{k}^{4} f\right\| \leq \sum_{j=0}^{3}\left\|B_{k}^{j}\left(B_{k} f-f\right)\right\| \leq 4\left\|B_{k} f-f\right\| .
$$

Theorem 4.1 with $\nu=0$ and $r=4$ repeated 4 times yields, for $g \in C^{4}$,

$$
\operatorname{Sup}_{\xi \in V_{S}}\left\|\varphi_{\xi}^{4}(\partial / \partial \xi)^{4} B_{n}^{4} g\right\| \leq \operatorname{Sup}_{\xi \in V_{S}}\left\|\varphi_{\xi}^{4}(\partial / \partial \xi)^{4} g\right\|
$$


Theorem 4.1 with $\nu=1$, used with $r=3$, then $r=2$, etc. yields

$$
\operatorname{Sup}_{\xi \in V_{S}}\left\|\varphi_{\xi}^{4}(\partial / \partial \xi)^{4} B_{k}^{4} f\right\| \leq C k^{2}\|f\| \text {. }
$$

A combination of (6.10), (6.11) and (6.12) yields

$$
K_{4, S}\left(f, t^{4}\right) \leq M\left(\left\|f-B_{k} f\right\|+t^{4} k^{2} K_{4, S}\left(f, k^{-1 / 2}\right)\right) .
$$

Following the inequality (6.13), we use a technique by V. Totik [15] used also in Theorem 9.3.4 of [11] and in $\S 5$ of [3] to obtain

$$
K_{4, S}\left(f, t^{4}\right) \leq C t^{\rho}\left(\sum_{1 \leq k \leq t^{-2}} k^{(\rho / 2)-1}\left\|B_{k} f-f\right\|+\|f\|\right)
$$

with any $\rho$ satisfying $0<\rho<4$.

We now substitute (6.14) in the Marchaud type estimate (3.16) with $r=3$ to obtain, with $3<\rho<4$,

$$
\begin{aligned}
K_{3, S}\left(f, t^{3}\right) \leq & C_{1} t^{3}\left(\sum_{1 \leq k \leq 1 / t} k^{2} k^{-\rho} \sum_{1 \leq l \leq k^{2}} l^{(\rho / 2)-1}\left\|B_{l} f-f\right\|\right) \\
& +C_{1} t^{3}\|f\| \\
\leq & C_{1} t^{3} \sum_{1 \leq l \leq 1 / t^{2}} l^{(\rho / 2)-1}\left\|B_{l} f-f\right\| \sum_{k \geq \sqrt{l}}^{\infty} k^{2-\rho}+C_{1} t^{3}\|f\| \\
\leq & C_{2} t^{3}\left(\sum_{1 \leq l \leq 1 / t^{2}} l^{1 / 2}\left\|B_{l} f-f\right\|+\|f\|\right) .
\end{aligned}
$$

For a given $n$, we choose $n_{0}$ satisfying $n / 2<n_{0} \leq n$ such that

$$
\left\|B_{n_{0}} f-f\right\|=\min _{n / 2<k \leq n}\left\|B_{k} f-f\right\| \text {. }
$$

For $P_{m} \in \Pi_{m},\left\|P_{m}-f\right\|=E_{m}(f)$ and $m=\left[\sqrt{n_{0}}\right]$, we have

$$
\begin{aligned}
K_{S}\left(f, m^{-1}\right) \leq & \left\|f-P_{m}\right\|+m^{-2}\left\|P(D) P_{m}\right\| \\
& +m^{-3} \max _{\xi \in V_{S}}\left\|\varphi_{\xi}^{3}(\partial / \partial \xi)^{3} P_{m}\right\| .
\end{aligned}
$$

We use (3.8) with $m$ and prove

$$
m^{-3} \max _{\xi \in V_{S}}\left\|\varphi_{\xi}^{3}(\partial / \partial \xi)^{3} P_{m}\right\| \leq C\left(K_{3, S}\left(f, m^{-3}\right)+m^{-3}\|f\|\right)
$$

and

(6.16) $m^{-2}\left\|P(D) P_{m}\right\| \leq C\left(K_{3, S}\left(f, m^{-3}\right)+m^{-3}\|f\|\right)+\left\|B_{n_{0}} f-f\right\|$. 
We now use the estimate of $K_{3, S}(f, t)$ for $t=m^{-1}$ and the inequalities (6.15) and (6.16) to obtain

$$
\begin{aligned}
& K_{S}\left(f, n^{-1 / 2}\right) \leq K_{S}\left(f, m^{-1}\right) \\
& \quad \leq\left\|B_{n_{0}} f-f\right\|+C m^{-3} \sum_{1 \leq l \leq n_{0}} l^{1 / 2}\left\|B_{l} f-f\right\|+C m^{-3}\|f\| \\
& \quad \leq C_{1}\left(n^{-3 / 2} \sum_{1 \leq l \leq n} l^{1 / 2}\left\|B_{l} f-f\right\|+n^{-3 / 2}\|f\|\right) .
\end{aligned}
$$

As $K_{S}\left(f-P_{1}, t\right)=K_{S}(f, t)$ and $E_{1}(f)=\left\|f-P_{1}\right\| \leq\left\|B_{1} f-f\right\|$, the term $\|f\|$ on the right-hand side of the above is redundant. Hence, we only have to show (6.15) and (6.16). To prove (6.16), we write, using (6.1) for $n_{0}\left(m=\left[\sqrt{n_{0}}\right]\right)$,

$$
\frac{1}{m^{2}}\left\|P(D) P_{m}\right\| \leq C\left(K_{3, S}\left(f, m^{-3}\right)+m^{-3}\|f\|\right)+\left\|B_{n_{0}} P_{m}-P_{m}\right\|
$$

We now estimate $\left\|B_{n_{0}} P_{m}-P_{m}\right\|$ by

$$
\begin{aligned}
\left\|B_{n_{0}} P_{m}-P_{m}\right\| & \leq\left\|B_{n_{0}} f-f\right\|+2 E_{m}(f) \\
& \leq\left\|B_{n_{0}} f-f\right\|+C\left(K_{3, S}\left(f, m^{-3}\right)+m^{-3}\|f\|\right)
\end{aligned}
$$

which completes the proof of (6.16). To prove (6.15), we follow the proof of Theorem 7.3.1 of [11] almost verbatim to show that

$$
\left\|\varphi_{\xi}^{4}(\partial / \partial \xi)^{4} P_{m}\right\| \leq L m^{4}\left(K_{3, S}\left(f, m^{-3}\right)+m^{-3}\|f\|\right)
$$

We then observe that it is sufficient to examine $\xi=e_{1}$. The rest follows from the one-dimensional theorem applied to $x_{1}$ at the coordinates $\left(x_{2}, \ldots, x_{d}\right)=\tilde{x}$. We obtain, using the mapping $y=x_{1} / 1-|\tilde{x}|$ and the notations $\varphi(y)^{2}=y(1-y), f_{\tilde{x}}(y)=f\left(x_{1}, x_{2}, \ldots, x_{d}\right)$ and $P_{m, \tilde{x}}(y)=P_{m}\left(x_{1}, x_{2}, \ldots, x_{d}\right)$,

$$
\begin{gathered}
\operatorname{Sup}_{0 \leq x_{1} \leq 1-|\tilde{x}|}\left|\varphi_{e_{1}}\left(x_{1}, \tilde{x}\right)^{r}\left(\partial / \partial x_{1}\right)^{r} P_{m}\left(x_{1}, \tilde{x}\right)\right| \\
=\operatorname{Sup}_{0 \leq y \leq 1}\left|\varphi(y)^{r}(\partial / \partial y)^{r} P_{m, \tilde{x}}(y)\right|
\end{gathered}
$$


and hence

$$
\begin{aligned}
& \operatorname{Sup}_{0 \leq x_{1} \leq 1-|\tilde{x}|}\left|\varphi_{e_{1}}\left(x_{1}, \tilde{x}\right)^{3}\left(\partial / \partial x_{1}\right)^{3} P_{m}(x, \tilde{x})\right| \\
& \quad=\operatorname{Sup}_{0 \leq y \leq 1}\left|\varphi(y)^{3}(\partial / \partial y)^{3} P_{m, \tilde{x}}(y)\right| \\
& \quad \leq C\left(m^{3} \operatorname{Sup}_{0<h \leq 1 / m}\left|\Delta_{h \varphi}^{3} f_{\tilde{x}}(y)\right|\right. \\
& \left.\quad+m^{-1} \operatorname{Sup}_{0 \leq y \leq 1}\left|\varphi(y)^{4}(\partial / \partial y)^{4} P_{m, \tilde{x}}(y)\right|\right) \\
& \leq C m^{3} \omega_{S}^{3}(f, 1 / m)+m^{-1} \operatorname{Sup}_{\xi \in V_{s}}\left\|\varphi_{\xi}^{4}(\partial / \partial \xi)^{4} P_{m}\right\|
\end{aligned}
$$

which, together with (6.17), completes the proof of (6.15). This in turn, completes the proof of (2.7).

7. The proof of the main results for $\bar{B}_{n}$. In this section, we point out some changes that are needed for the proof of the saturation, direct and converse results for $\bar{B}_{n}$, that is, the proof of Theorem 2.2. We first need a form of Theorem 3.1 and Corollary 3.2 on the cube whose proof is simpler.

Theorem 7.1. For the cube $Q$ given in (1.4), $0<p \leq \infty$ and $\xi \in V_{Q}$, we have, for $P \in \Pi_{n}$,

$$
\begin{aligned}
& \left\|w(\cdot) \tilde{d}\left(Q, \xi_{1}, \cdot\right)^{1 / 2} \cdots \tilde{d}\left(Q, \xi_{k}, \cdot\right)^{1 / 2} \frac{\partial}{\partial \xi_{1}} \cdots \frac{\partial}{\partial \xi_{k}} P\right\|_{L_{p}(Q)} \\
& \quad \leq C n^{k}\|w P\|_{L_{p}(Q)}
\end{aligned}
$$

where $w(x) \equiv x_{1}^{\alpha_{1}} \cdots x_{d}^{\alpha_{d}}\left(1-x_{1}\right)^{\beta_{1}} \cdots\left(1-x_{d}\right)^{\beta_{d}}, \alpha_{i}, \beta_{i} \geq 0$ for $p=\infty$ and $\alpha_{i}, \beta_{i}>1 / p$ for $p<\infty$.

We note that the rest of the results in $\S 3$ are for a simple polytope $S$ which applies to $Q$ as a special case.

The analogues of Theorems 4.1 and 5.1 follow easily. In the statements, we replace $S$ by $Q$. We do not need the elaborate scheme of dividing $Q$ into subdomains and using a transformation like $T$. In fact, we treat the whole domain $Q$ in the same way as we treated $V_{0}$ in Theorems 4.1 and 5.1. We observe that $\bar{B}_{n}(f, x)$ is a polynomial of degree $n d$ (not $n$ ) but if $P \in \Pi_{m \leq n}, \bar{B}_{n}(P, x)$ is a polynomial of degree $m$. We further note that in the analogue of Lemma 5.2, (e), (f) and (g) are changed in the following manner. 
LEMMA 7.2. For $\psi_{i}(x)=x_{i}, i=1, \ldots, d$, (a), (b), (c) and (d) of Lemma 5.2 hold with $\bar{B}_{n}$ replacing $B_{n}$. Furthermore, we have

$(\mathrm{e})^{\prime} \bar{B}_{n}\left(\left(\psi_{i}-x_{i}\right)\left(\psi_{j}-x_{j}\right), x\right)=0$ for $i \neq j$, and

$(\mathrm{f})^{\prime} \bar{B}_{n}\left(\left(\psi_{i}-x_{i}\right)\left(\psi_{j}-x_{j}\right)\left(\psi_{l}-x_{l}\right), x\right)=0$ for $i \neq j \neq l \neq i$,

$$
(\mathrm{g})^{\prime} \bar{B}_{n}\left(\left(\psi_{i}-x_{i}\right)\left(\psi_{j}-x_{j}\right)^{2}, x\right)=0 \text { for } i \neq j \text {. }
$$

Theorem 6.1 for $\bar{B}_{n}$ follows verbatim and the same is true for the rest of $\S 6$.

8. Comparisons, further questions and conjectures. When the results in [7], [8] and [11] are examined, it is clear that, for $0<\alpha<2$,

$$
\begin{aligned}
\left\|B_{n} f-f\right\|_{C(S)}=O\left(n^{-\alpha / 2}\right) & \Leftrightarrow K_{2, S}\left(f, t^{2}\right)_{\infty}=O\left(t^{\alpha}\right) \\
& \Leftrightarrow E_{n}(f)_{C(S)}=O\left(n^{-\alpha}\right) .
\end{aligned}
$$

In [2], the investigation of $K_{2, S}\left(f, t^{2}\right)_{\infty}$ (in our notation) is pursued but, in view of the present results, there is no hope to include saturation in the context of $K_{2, S}\left(f, t^{2}\right)_{\infty}$.

For $K_{S}(f, t)$, given in (3.7),

$$
K_{S}(f, t) \geq K_{3, S}\left(f, t^{3}\right) .
$$

As Theorem 3.4 yields, for $\alpha<2$, the implication

$$
K_{3, S}\left(f, t^{3}\right)=O\left(t^{\alpha}\right) \quad \text { implies } K_{2, S}\left(f, t^{2}\right)=O\left(t^{\alpha}\right),
$$

we also have (for $\alpha<2$ )

$$
K_{3, S}\left(f, t^{3}\right)=O\left(t^{\alpha}\right) \text { implies } K_{S}(f, t)=O\left(t^{\alpha}\right) .
$$

It is now clear that $K_{3, S}\left(f, t^{3}\right)$ and $K_{S}(f, t)$ can replace $K_{2, S}\left(f, t^{2}\right)$ in (8.1). Of course, (8.1) does not include results at the saturation rate $n^{-1}$ or close to it as $n^{-1}(\log n)^{\alpha}$ or $n^{-1}(\log n)^{\alpha}(\log \log n)^{\beta}$ for example. One may be led to believe that the behaviour of $K_{3, s}\left(f, t^{3}\right)$ is sufficient. However, it fails already in the univariate case.

To concentrate on possible generalization, we define

$$
K_{S}^{*}\left(f, t^{2}\right)_{\infty}=\operatorname{Inf}_{g \in C^{2}(S)}\left(\|f-g\|+t^{2}\|P(D) g\|\right)
$$

with $P(D)$ of (3.2). Now, we may ask if a result utilizing $K_{S}^{*}\left(f, t^{2}\right)_{\infty}$ which contains saturation, direct and converse theorems is possible. We believe that the answer will be affirmative. (If the requirement that the saturation theorem will be included is dropped, the present technique and discussion is sufficient.) 
We believe that an even stronger result is valid. We conjecture that a strong converse inequality of type $A$ in the terminology of [10], which will imply that

$$
\left\|B_{n} f-f\right\| \sim K_{S}^{*}\left(f, n^{-1}\right)_{\infty},
$$

will be proved one day. We recall that even for the univariate Bernstein polynomial, (8.3) is open. For $d>1$, even the weakest "strong converse inequality of type $D$ " (in the terminology of [10]), that is,

$$
\operatorname{Sup}_{k \geq n}\left\|B_{k} f-f\right\| \sim K_{S}^{*}\left(f, n^{-1}\right)
$$

is not known. However, for Bernstein-Durrmeyer operators (see [4]), the analogue of (8.3) is known for $1<p<\infty$ and all dimensions $d$ (and for $d=1,2,3$ for $1 \leq p \leq \infty$ ). There are some possible results between (8.3) and the result of this paper, but as we are in the business of making conjectures, we might as well be brave.

Another interesting question is to find out the actual behaviour of $f$ from the $K$-functional or from the rate of convergence. Here, results in the multivariate case are very scant. We conjecture that

$$
\|P(D) f\| \leq M \quad \text { implies }\left\|h^{-2} \Delta_{h \varphi_{\xi} \xi}^{3} f\right\| \leq M_{1} \quad \text { for } \xi \in V_{S}
$$

where $M_{1}$ depends on $M$ but not on $h$ or $f$.

\section{REFERENCES}

[1] H. Berens and Y. Xu, Bernstein-Durrmeyer polynomials with Jacobi weights, Approximation Theory and Functional Analysis, C. K. Chui ed., Academic Press, 1990, 25-46.

[2] _ K- K-moduli, moduli of smoothness, and Bernstein polynomials on simplices, (manuscript).

[3] W. Chen and Z. Ditzian, Best polynomial and Durrmeyer approximation in $L_{p}(S)$, Indag. Math. (N.S.), 2 (1991), 437-452.

[4] W. Chen, Z. Ditzian and K. Ivanov, Strong converse inequality for the BernsteinDurrmeyer operator, J. Approx. Theory, (to appear).

[5] Z. Ditzian, A global inverse theorem for combinations of Bernstein polynomials, J. Approx. Theory, 26 (1979), 277-292.

[6] - Saturation of approximation of $n$-dimensional functions, Constructive function theory, Proceedings of conference held in Varna, Bulgaria, 1981, Sofia, Bulgaria, 1983, pp. 295-300.

[7] _ Inverse theorems for multi-dimensional Bernstein operators, Pacific J. Math., 121 (1986), 293-319.

[8] _ Best polynomial approximation and Bernstein polynomial approximation on a simplex, Indag. Math., 92 (1989), 243-256.

[9] _ Multivariate Bernstein and Markov inequalities, J. Approx. Theory, 70 (1992), 273-283. 
[10] Z. Ditzian and K. Ivanov, Strong converse inequality, J. d'Analise Mat., (to appear).

[11] Z. Ditzian and V. Totik, Moduli of Smoothness, Springer-Verlag, 1987.

[12] Z. Ditzian and X. Zhou, Kantorovich-Bernstein polynomials, Constructive Approximation, 6 (1990), 421-435.

[13] R. Q. Jia and Z. C. Wu, Bernstein polynomial on simplices (Chinese), Acta Math. Sinica, 31 (1988), 510-522.

[14] P. G. Nevai, Bernstein inequality in $L^{p}, 0<p<1$, J. Approx. Theory, 27 (1979), 239-243.

[15] V. Totik, An interpolation theorem and its applications to positive operators, Pacific J. Math., 111 (1984), 447-481.

Received June 17, 1991. The first author was supported by NSERC grant A-4816 of Canada.

UNIVERSITY OF ALBERTA

Edmonton, Alberta, Canada T6G 2G1

AND

UNIVERSITY OF DUISBURG

Duisburg, Germany D 4100 


\title{
PACIFIC JOURNAL OF MATHEMATICS
}

Founded by

\author{
E. F. BeCKeNBACH (1906-1982) F. Wolf (1904-1989)
}

\section{EDITORS}

\section{S. VARADARAJAN (Managing Editor) University of California Los Angeles, CA 90024-1555 vsv@math.ucla.edu \\ F. Michael Christ University of California Los Angeles, CA 90024-1555 christ@math.ucla.edu}

\section{Herbert Clemens}

University of Utah

Salt Lake City, UT 84112

clemens@math.utah.edu

\author{
THOMAS ENRIGHT \\ University of California, San Diego \\ La Jolla, CA 92093 \\ tenright@ucsd.edu \\ Nicholas ERcolani \\ University of Arizona \\ Tucson, AZ 85721 \\ ercolani@math.arizona.edu \\ R. FINN \\ Stanford University \\ Stanford, CA 94305 \\ finn@gauss.stanford.edu \\ VAUGHAN F. R. JONES \\ University of California \\ Berkeley, CA 94720 \\ vfr@math.berkeley.edu
}

\section{SUPPORTING INSTITUTIONS}

UNIVERSITY OF ARIZONA

UNIVERSITY OF BRITISH COLUMBIA

UNIVERSITY OF OREGON

CALIFORNIA INSTITUTE OF TECHNOLOGY

UNIVERSITY OF CALIFORNIA

UNIVERSITY OF MONTANA

UNIVERSITY OF NEVADA, RENO

NEW MEXICO STATE UNIVERSITY

OREGON STATE UNIVERSITY
SteVen KerckhofF

Stanford University

Stanford, CA 94305

spk@gauss.stanford.edu

MARTIN SCHARLEMANN

University of California

Santa Barbara, CA 93106

mgscharl@henri.ucsb.edu

HAROLD STARK

University of California, San Diego

La Jolla, CA 92093

\author{
STANFORD UNIVERSITY \\ UNIVERSITY OF HAWAII \\ UNIVERSITY OF UTAH \\ WASHINGTON STATE UNIVERSITY \\ UNIVERSITY OF WASHINGTON
}

UIV 


\section{PACIFIC JOURNAL OF MATHEMATICS}

Volume $158 \quad$ No. $1 \quad$ March 1993

Determinant identities

GEORGE W. Eyre ANDrEWS and WiLliam H. Burge

A spectral theory for solvable Lie algebras of operators

E. BOASSO and ANGEL RAFAEL LAROTONDA

Simple group actions on hyperbolic Riemann surfaces of least area

S. Allen Broughton

Duality for finite bipartite graphs (with an application to $\mathrm{II}_{1}$ factors)

MARIE CHODA

Szegő maps and highest weight representations

MARK GREGORY DAVIDSON and Ron STANKE

Optimal approximation class for multivariate Bernstein operators

ZEEV DitZIAN and XINLONG ZHOU

Witt rings under odd degree extensions

ROBERT FITZGERALD

Congruence properties of functions related to the partition function

ANTHONY D. FORBES

Bilinear operators on $L^{\infty}(G)$ of locally compact groups

Colin C. GRAham and Anthony To-Ming LAU

Nonuniqueness of the metric in Lorentzian manifolds

GEOFFREY K. MARTIN and GERARD THOMPSON

Index theory and Toeplitz algebras on one-parameter subgroups of Lie 189 groups

EFTON PARK 